\title{
Fibroelastoma Recurrence in Left Ventricle: Rarity of Primary Cardiac Tumor
}

\author{
Inna Kammerer ${ }^{1}$ Reinhard Besser ${ }^{1}$ Mohammed Al-Azani ${ }^{1} \quad$ Christian Frank ${ }^{1}$ Manfred H. Bohrer ${ }^{2}$ \\ Falk-Udo Sack ${ }^{1}$ \\ ${ }^{1}$ Department of Cardiac Surgery, Hospital of City Ludwigshafen, \\ Ludwigshafen, Germany \\ 2 Institute of Pathology, Hospital of City Ludwigshafen, Ludwigshafen, \\ Germany \\ Address for correspondence Inna Kammerer, MD, Department of \\ Cardiac Surgery, Hospital of City Ludwigshafen, Bremserstreet 79, D- \\ 67063 Ludwigshafen, Germany (e-mail: kammerei@klilu.de).
}

Surg J 2015;1:e35-e37.
Abstract
Keywords
- left ventricular fibroelastoma recurrence
- left ventricular cardiac tumor
- transesophageal echocardiography

Cardiac papillary fibroelastoma (CPF) is a primary cardiac neoplasm usually detected by echocardiography. Left ventricular fibroelastomas are extremely rare. The incidence of CPF is between 0.0017 and $0.33 \%$ during autopsy studies. We report a 70 -year-old man who had papillary fibroelastoma discovered and resected in 2005 that recurred in 2013. The tumor grew rapidly from 2013 to 2014 . A bioprosthetic mitral valve was placed in 2014. Due to the location and nature of the recurrent tumor, mitral valve replacement was the treatment of choice to prevent a third recurrence of the fibroelastoma. The patient was discharged from the hospital on postoperative day 9 .

Cardiac papillary fibroelastoma is a primary cardiac neoplasm with an unknown prevalence but is increasingly detected by echocardiography.

A 70-year-old asymptomatic man $(98 \mathrm{~kg} ; 178 \mathrm{~cm})$ was referred in 2014 to our hospital for evaluation of two suspected cardiac tumors in the left ventricle after his first open heart surgery for fibroelastoma via conservative sternotomy in year 2005. The patient presented to our hospital with no neurologic, cardiac, or other signs or symptoms of general discomfort before he was scheduled for surgery.

In 2005, after a singular transient ischemic event, a cardiac tumor was confirmed by transesophageal echocardiography in the left ventricle intertwined in the papillary muscle belly close to the mitral valve cusp. The tumor was surgically resected through a median sternotomy with the mitral valve preserved. At that time, no visible residues of the fibroelastoma had been left macroscopically, and no clinical complications in the surgical and postoperative course were then observed. During the surgery in 2005, the tumor was found on the A1 segment of the anterior leaflet and on the posterior chordae in two parts. The tumor was resected with visual

received

June 13, 2015 accepted after revision September 4, 2015 published online November 5, 2015 confirmation. The histologic evaluation indicated a primary cardiac papillary growth with a pale basophilic myxoid stroma, with nuclear matrix eosinophilic without neoplasm potency (-Fig. 1A). Annual echocardiography and regular blood tests showed no fibroelastoma present or mitral valve dysfunction.

Three years following removal of the fibroelastoma, a small formation of the tumor was detected by echocardiography. The clinical course was uneventful; therefore, no special treatment was indicated at that time. From 2013 to 2014, the tumor rapidly grew, and the patient developed benign prostate hyperplasia, which was treated with Doudart $0.4 \mathrm{mg}$ (dutasteride + tamsulosin; GlaxoSmithKline, London, United Kingdom).

In 2014, the fibroelastoma recurred as detected by transesophageal echocardiography. The function of the mitral valve was normal, but the tumor tissue was detected between the papillary muscle and the chordae tendineae. Magnetic resonance imaging revealed two separate mobile and noncalcified spherical structures $(1 \times 0.8 \mathrm{~cm})$, attached to the ventricular surface of the posterior leaflet of the mitral valve with
Copyright $\odot 2015$ by Thieme Medical Publishers, Inc., 333 Seventh Avenue, New York, NY 10001, USA. Tel: +1(212) 584-4662.
License terms

10.1055/s-0035-1565244. ISSN 2378-5128.

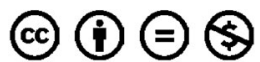


A First tumor (year 2005)

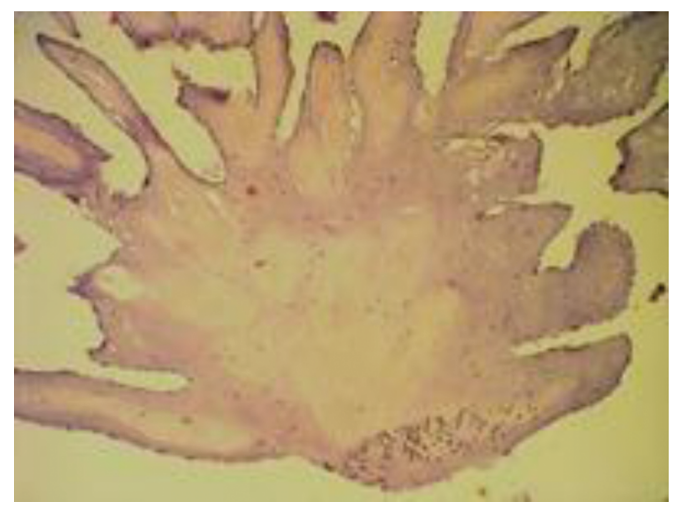

B New fibroelastoma (2014)

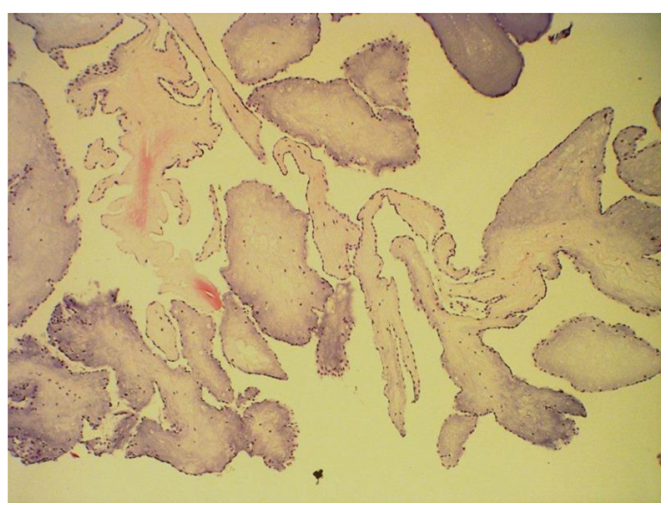

Fig. 1 Histologic results of fibroelastoma in left ventricular papillary muscle. (A) First tumor (2005). (B) New fibroelastoma (2014).

intermediate signal intensity on a steady-state free precession sequence and high signal on T2-weighted sequences. Clinically, the patient presented with New York Heart Association class $\leq$ II without any cardiac or neurologic symptoms at the time.

\section{Operative Setting}

The operation was performed through a median redo sternotomy with mild hypothermia and cardiopulmonary bypass with anterograde cold crystalloid cardioplegia provided by 1,500 mL of Custodiol (histidine-tryptophan-ketoglutarate; Koehler Chemie, Bensheim, Germany). The inspection of the mitral valve through left transatrial aperture showed formation of two solid tumors interwoven tightly in the papillary muscle bellies and chordae (-Fig. 2 ).

A mitral valve reconstruction attempt was avoided due to the unsuccessful initial surgical elimination of tumor, which led to the recurrence of the fibroelastoma. The repeated histologic evaluation confirmed the imaging diagnosis hypothesis of the fibroelastoma recurrence $(6.5 \times 3.5 \times 0.7 \mathrm{~cm}$ and $1.5 \times 1.2 \times 0.7 \mathrm{~cm}$; - Fig. 1B).

The mitral annulus morphology allowed for implantation of a 33-mm Hancock II (Medtronic, Inc., Dublin, Ireland) biological prosthesis for advanced age ( $>70$ years) at the request of the patient. The prosthesis was implanted in a typical manner using pledged 2-0 Ethibond sutures (Ethicon, Inc., Somerville, New Jersey, United States). Once the sutures were knotted, it was important to verify proper placement of the biological valve, to avoid paravalvular leaks. The left atrium was closed in the usual manner, double-layered with 4-0 polypropylene monofilament. Anticoagulation therapy (vitamin $\mathrm{K}$ antagonist) was required for 3 months after the mitral valve replacement.

The cross-clamping time was 55 minutes; the total cardiopulmonary bypass time was 75 minutes. The patient was transferred to the intensive care unit and was extubated after 3.7 hours; time in the intensive care unit was $<24$ hours. Postoperatively, the patient was discharged after 9 days in excellent physical and mental health. The postoperative transesophageal echocardiography showed the correct position and proper function of the prosthesis without any unusual technical or morphological errors. Postoperatively, this patient showed no episodes of arrhythmia or neurologic adverse effects.

\section{Discussion}

The incidence of primary cardiac tumors is very rare $(0.0017$ to $0.33 \%$ ) at autopsy and forensic pathology studies. ${ }^{1-3}$ The fibroelastoma consisted of a small papillary, pedunculated, and avascular tumor, covered by a single layer of endothelium containing variable amounts of elastic fibrils in the form of composed collagenous connective tissue in hyaline stroma. ${ }^{4}$ The fibroelastoma tumors are typically located in more than $95 \%$ of cases in the left ventricle. In $45 \%$ of these cases, the tumor is positioned on the aortic valve, followed by mitral (36.4\%) and pulmonary valve ( $8 \%)^{1}{ }^{1}$

The hypothetical reasons for tumor development include the hemodynamic stress of blood flow along the endocardium following endothelial damage and a possible disposal of fibrin fibers that are used to form organized thrombus. Patients with fibroelastomas who undergo surgical treatment tend to have large tumors with no infiltration of the surrounding tissue.

Usually the fibroelastoma tumors that are resected in patients who have undergone surgical treatment do not infiltrate the surrounding cardiac tissue. The relapse of fibroelastoma was never described because it is a benign neoplasm with no propensity to recur. The tumor in our case would not have been found without critical evaluation of our patient's clinical case history when changing drug and surgical treatment for another disease.

Surgical treatment is indicated in cases of neurologic manifestations and in hospitalized patients suffering from syncope, transient ischemic event, or atrial fibrillation following diagnostic imaging. ${ }^{4,5}$ Surgery is also indicated after percutaneous persistent foramen oval closure, ${ }^{6}$ or chest pain with ST-segment elevation as myocardial infarction concerning the location of the fibroelastoma on the right or left coronary cusp. But long-term follow-up studies using echocardiography for imaging have not been done yet, and therefore the natural history or clinical manifestation of these 


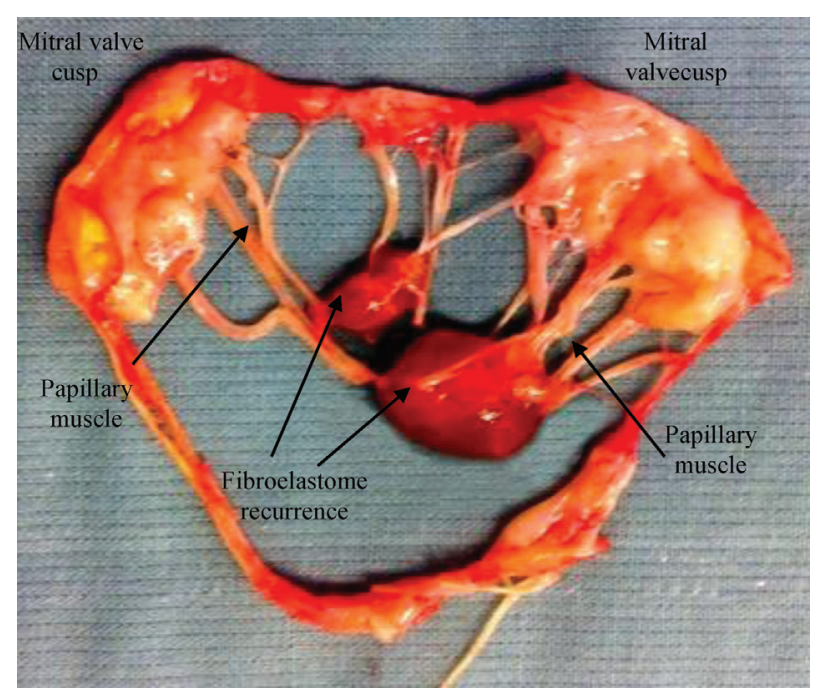

Fig. 2 Fibroelastoma recurrence on the mitral valve in vitro (year 2014).

tumors has not been presented except in one non-Hodgkin disease case (of 23 patients) within 15 years follow-up. ${ }^{7}$

The surgical resection of fibroelastoma has a success rate of $83 \%$ with the valves being preserved and is classified as a curative, safe, and well-tolerated procedure. ${ }^{8-11}$ The matrix consists of elastic fibers, proteoglycans, and spindle cells that resemble smooth muscle cells or fibroblasts. The tumor connective tissue contains longitudinally oriented collagen with irregular elastic fibers. ${ }^{12}$

In our case, the mitral valve was replaced to prevent recurrence of the fibroelastoma and a third operation on this patient. The mitral valve apparatus was excised and replaced with a biological prosthesis. Three criteria in the decision-making process to perform mitral valve replacement instead of tumor resection alone were crucial.

1. Because the fibroelastomas were adherent to almost all chordae and to the papillary muscles, the removal of the tumors required the resection of too much material of the subvalvular mitral valve apparatus including many chordae (-Fig. 2). Furthermore, the chordae were very thin, which would complicate a proper surgical resection by using many neochordae. The preservation of the mitral valve apparatus seemed unreasonable, and successful reconstruction of the mitral valve would be unlikely and unreliable.

2. During the first surgery for the removal of the fibroelastoma from the chordate macroscopically, no remnants were seen. It was unlikely that all microscopic particles were removed (especially those growing on the chordae) of the fibroelastoma. Our study showed a recurrence of the fibroelastoma years later.

3. Considering the age of the patient, the probability of the mitral valve reconstruction being complicated and unsuccessful, and the risk of recurrence of the tumor making surgery again necessary, the clinical safety and efficacy for the patient was paramount.

\section{Conclusion}

Cardiac fibroelastomas are very rare, and their presence is often found in patients with echocardiography by chance or after a neurologic event. In our case, routine follow-up detected the recurrence of the tumor without any serious incident. To our knowledge, there has been no published report on fibroelastoma recurrence in a cardiac cohort. The surgical treatment with a biological mitral valve replacement was indicated as a safe alternative to mitral valve repair to avoid oral anticoagulation therapy in an elderly patient.

\section{Disclosures \\ None}

\section{References}

1 Generali T, Tessitore G, Mushtaq S, Alamanni F. Pulmonary valve papillary fibroelastoma: management of an unusual, tricky pathology. Interact Cardiovasc Thorac Surg 2013;16(1):88-90

2 Meng F, Sun JP, Chen M, et al. An unusual cardiac fibroelastoma case. Circulation 2014;130(6):520-522

3 Elbardissi AW, Dearani JA, Daly RC, et al. Survival after resection of primary cardiac tumors: a 48-year experience. Circulation 2008; 118(14, Suppl):S7-S15

4 Mathis S, Lamy M, Ciron J, et al. Paroxysmal sneezing at the onset of syncopes and transient ischemic attack revealing a papillary cardiac fibroelastoma. Case Rep Neurol Med 2014; 2014:734849

5 Gowda RM, Khan IA, Nair CK, Mehta NJ, Vasavada BC, Sacchi TJ. Cardiac papillary fibroelastoma: a comprehensive analysis of 725 cases. Am Heart J 2003;146(3):404-410

6 Vaskelyte L, Risteski P, Bertog S, Hofmann I, Hartmann S, Sievert H. A case of a fibroelastoma and patent foramen ovale in a patient with prior stroke. Cardiovasc Diagn Ther 2014;4(1):47-49

7 Anastacio MM, Moon MR, Damiano RJJr, Pasque MK, Maniar HS, Lawton JS. Surgical experience with cardiac papillary fibroelastoma over a 15-year period. Ann Thorac Surg 2012;94(2): 537-541

8 Aryal MR, Badal M, Mainali NR, Jalota L, Pradhan R. Papillary fibroelastoma of the aortic valve: an unusual cause of angina. World J Cardiol 2013;5(4):102-105

9 Dias RR, Fernandes F, Ramires FJ, Mady C, Albuquerque CP, Jatene FB. Mortality and embolic potential of cardiac tumors. Arq Bras Cardiol 2014;103(1):13-18

10 Sun JP, Asher CR, Yang XS, et al. Clinical and echocardiographic characteristics of papillary fibroelastomas: a retrospective and prospective study in 162 patients. Circulation 2001;103(22): 2687-2693

11 Sato M, Nagaya K, Hatakeyama M, Komatsu T. Multiple papillary fibroelastoma: report of a case and implications for management. Gen Thorac Cardiovasc Surg 2014;62(2):122-124

12 Agaimy A, Rösch J, Weyand M, Strecker T. Primary and metastatic cardiac sarcomas: a 12-year experience at a German heart center. Int J Clin Exp Pathol 2012;5(9):928-938 